\title{
Flaxseed oil ameliorates alcoholic liver disease via anti-inflammation and modulating gut microbiota in mice
}

\author{
Xiaoxia Zhang ${ }^{1,2}$, Hao Wang ${ }^{2}$, Peipei Yin ${ }^{1}$, Hang Fan ${ }^{1}$, Liwei Sun ${ }^{1}$ and Yujun Liu*
}

\begin{abstract}
Background: Alcoholic liver disease (ALD) represents a chronic wide-spectrum of liver injury caused by consistently excessive alcohol intake. Few satisfactory advances have been made in management of ALD. Thus, novel and more practical treatment options are urgently needed. Flaxseed oil (FO) is rich in a-linolenic acid (ALA), a plant-derived n-3 polyunsaturated fatty acids (PUFAs). However, the impact of dietary FO on chronic alcohol consumption remains unknown.

Methods: In this study, we assessed possible effects of dietary FO on attenuation of ALD and associated mechanisms in mice. Firstly, mice were randomly allocated into four groups: pair-fed (PF) with corn oil (CO) group (PF/CO); alcohol-fed (AF) with CO group (AF/CO); PF with FO group (PF/FO); AF with FO group (AF/FO). Each group was fed modified Lieber-DeCarli liquid diets containing isocaloric maltose dextrin a control or alcohol with corn oil and flaxseed oil, respectively. After 6 weeks feeding, mice were euthanized and associated indications were investigated.

Results: Body weight (BW) was significantly elevated in AF/FO group compared with AF/CO group. Dietary FO reduced the abnormal elevated aspartate aminotransferase (AST) and alanine aminotransferase (ALT) levels in chronic ethanol consumption. Amelioration of these parameters as well as liver injury via HE staining in dietary FO supplementation in ALD demonstrated that dietary FO can effectively benefit for the protection against ALD. To further understand the underlying mechanisms, we investigated the inflammatory cytokine levels and gut microbiota. A series of inflammatory cytokines, including TNF-a, IL-1 $\beta, I L-6$ and IL-10, were determined. As a result, TNF- $\alpha, I L-1 \beta$ and IL- 6 were decreased in AF/FO group compared with control group; IL-10 showed no significant alteration between AF/CO and AF/FO groups ( $p>0.05$ ). Sequencing and analysis of gut microbiota gene indicated that a reduction of Porphyromonadaceae and Parasutterella, as well as an increase in Firmicutes and Parabacteroides, were seen in AF group compared with PF control. Furthermore, dietary FO in ethanol consumption group induced a significant reduction in Proteobacteria and Porphyromonadaceae compared with AF/CO group.
\end{abstract}

Conclusion: Dietary FO ameliorates alcoholic liver disease via anti-inflammation and modulating gut microbiota, thus can potentially serve as an inexpensive interventions for the prevention and treatment of ALD.

Keywords: Flaxseed oil, ALD, Anti-inflammation, Gut microbiota

\footnotetext{
*Correspondence: yjliubio@bjfu.edu.cn

${ }^{1}$ College of Biological Sciences and Biotechnology, Beijing Forestry University,

Qinghua Donglu No35, Haidian District, Beijing 100083, China

Full list of author information is available at the end of the article
} 


\section{Background}

Alcoholic liver disease (ALD) represents a chronic widespectrum of liver injury caused by consistently excessive alcohol intake, ranking major causes of morbidity and mortality worldwide among people who abuse alcohol [1]. ALD includes a histological spectrum of liver injure ranging from simple steatosis to hepatitis characterized by inflammation, with potential progression to fibrosis and cirrhosis. Hepatitis, with an occurrence of approximately 10 to $35 \%$ in chronic drinkers and responsible for more than $1 / 3$ significant morbidity and mortality, has been thought to play a crucial role in reversible pathological process of ALD [2-4]. Up to now, few satisfactory advances have been made in management of ALD, except abstinence from alcohol $[4,5]$. Thus, novel and more practical treatment options are urgently needed.

Gut microbiota play a crucial role in progression and pathogenesis of ALD. Accumulating evidence has revealed that gut microbiota is closely associated with liver in ALD as the gut-liver axis [6,7]. Impairment of gut microbiota homeostasis in ALD induces proliferation of gram negative pathogenic bacteria, which generate lipopolysaccharide (LPS) and translocate to liver tissue as a trigger for hepatitis by binding to TLR-4 (Toll-like receptor-4) on macrophages and neutrophils. Moreover, Campos Canesso et al. showed that the administration of alcohol to germ-free mice is associated to the absence of liver inflammation and injury, indicating that alcohol alone is not sufficient for the development of liver disease, and that the presence of microbiota alterations is also necessary [8]. Thus, modulation of gut microbiota dysbiosis could attenuate hepatic injury in $\operatorname{ALD}[3,9]$.

Flaxseed oil (FO) is rich in plant-derived omega-3 (n-3) polyunsaturated fatty acids (PUFAs), mainly $\alpha$-linolenic acid (ALA, 18:3 n-3). Clinical studies reported that a low levels of $n-3 P U F A s$ in serum and liver tissue is a common characteristic of ALD patients [10,11]. Dietary FO prevented against acute alcoholic hepatic steatosis via ameliorating lipid homeostasis at adipose tissue-liver axis in mice [11]. However, the impact of dietary FO on inflammation and gut micorbiota in chronic ALD remains unknown.

In the present study, we assessed effects of dietary FO on attenuation of ALD and associated mechanisms in mice. Results of the study may contribute to understanding the role played by FO in ALD and the complexity of the interplay among the diet, gut microbiota, inflammation and ALD.

\section{Methods}

Animals and diet

Sixty male C57BL/6 J mice (8 weeks old) were obtained from Vital River Laboratory Animal Technology Co. Ltd., Beijing, China. The animals were housed in individual cages in a temperature-controlled $\left(22 \pm 1{ }^{\circ} \mathrm{C}\right)$, light-cycled (12-h light/dark cycle) room.

All liquid diets for mice feeding were purchased from TROPHIC Animal Feed High-tech Co., Ltd., Nantong, China.

\section{Experimental design}

After an 1-week period of acclimation to the control liquid diet, maleC57BL/6 J mice ( $n=60,8$ weeks old) were fed the modified Lieber-DeCarli liquid diets as previously described [11]. Briefly, mice were randomly allocated into four groups (15 animals/group): (a) pair-fed (PF) with corn oil $(\mathrm{CO})$ group $(\mathrm{PF} / \mathrm{CO})$, mice were fed modified LieberDeCarli CO liquid diets containing isocaloric maltose dextrin as $\mathrm{CO}$ control; (b) alcohol-fed (AF) with $\mathrm{CO}$ group $(\mathrm{AF} / \mathrm{CO})$, mice were fed ethanol-containing modified Lieber-DeCarli CO liquid diets; (c) PF with flaxseed oil (FO) group (PF/FO), mice were fed modified LieberDeCarli FO liquid diets containing isocaloric maltose dextrin as FO control; (d) AF with FO group (AF/FO), mice were fed ethanol-containing modified Lieber-DeCarli FO liquid diets. Mice in AF groups were fed the modified Lieber-DeCarli liquid diets containing ethanol with an energy composition of $18 \%$ protein, $19 \%$ carbohydrate, $35 \%$ fat and $28 \%$ ethanol, whereas animals in the PF groups were fed the modified Lieber-DeCarli liquid diets, in which, isocaloric maltose dextrin (carbohydrate) replaced ethanol, and $35 \%$ of the total calories were provided by either corn oil (rich in n-6 PUFAs) or flaxseed oil (rich in n3 PUFAs). Components of the liquid diets and the fatty acid composition of dietary fats are shown in Additional file 1 (Table S1) and Additional file 2 (Table S2), respectively. Groups (a) and (c) were the pair-fed controls for groups (b) and (d), respectively. Liquid diets were freshly prepared from powder daily according to the manufacturer's instruction. Average daily volume of liquid intake per mouse was monitored and calculated in AF groups. Mice in PF groups consume equal amounts of diets. After 6 weeks of feeding, mice were then euthanized and associated indications were investigated. Blood samples were collected in ethylene diamine tetraacetic acid (EDTA)-containing tubes and centrifuged $(1200 \times \mathrm{g}$ for $15 \mathrm{~min})$ to obtain plasma samples. All plasma samples were stored at $-80{ }^{\circ} \mathrm{C}$ for further analysis.

\section{Determination of plasma AST and ALT levels}

As biochemical indicators of liver function, plasma aspartate aminotransferase (AST) and alanine aminotransferase (ALT) activities in each group were respectively determined using AU400 automatic biochemical analyzer (Olympus, Japan). 


\section{Determination of plasma endotoxin}

Plasma LPS levels in each mouse/group were measured with limulus amebocyte lysate kit (Xiamen Bioendo Technology Co.Ltd, Xiamen, China) according to the manufacturer's instructions.

\section{HE staining}

After mice sacrifice, liver tissues were immediately fixed with formalin and processed with hematoxylin-eosin (HE) staining to evaluate liver damage including hepatocyte fatty change, inflammatory cells, degeneration and necrosis.

\section{ELISA assays}

Liver tissues $(0.5 \mathrm{~g})$ were homogenized in $1.5 \mathrm{ml}$ ice-cold $50 \mathrm{mM}$ Tris buffer (pH7.2, Tris with 1\% Triton-X 100 and $0.1 \%$ protease inhibitor) and shaken on ice for $90 \mathrm{~min}$. Then the homogenates were centrifuged at $3,000 \times \mathrm{g}$ for $15 \mathrm{~min}$. Supernatants were collected for determination of tumor necrosis factor (TNF)- $\alpha$, IL (interleukin)-1 $\beta$, IL-6 and IL-10 concentrations. Measurements of each cytokine level in plasma or the supernatants of liver tissues were performed by enzyme linked immunosorbent assay (ELISA) according to the manufacturer's instructions (e-Bioscience, CA, USA).

\section{Gut microbiota analysis}

The fecal microbial 16S rRNA gene sequencing and analysis were investigated as previously described [12]. After 6 weeks feeding, five mice per group were randomly selected and transferred to fresh sterilized cages. The fresh feces of each mouse was respectively collected, immediately frozen in liquid nitrogen, and then stored at $-80{ }^{\circ} \mathrm{C}$ until DNA extraction.

Microbial DNA was extracted from $200 \mathrm{mg}$ feces samples as previously described [13]. Briefly, this sample (200 mg) was resuspended in $4 \mathrm{ml}$ of $4 \mathrm{M}$ guanidine thiocyanate-0.1 M Tris (pH7.5) and $600 \mu \mathrm{l}$ of $10 \% \mathrm{~N}$ lauroyl sarcosine. The feces was ground with a mortar on ice, $250 \mu \mathrm{g}$ of the ground material was transferred to a 2-ml screw-cap polypropylene microcentrifuge tube, and the remaining material was frozen. After addition of $500 \mu \mathrm{l}$ of $5 \% \mathrm{~N}$-lauroyl sarcosine $0.1 \mathrm{M}$ phosphate buffer (pH8.0), the $2 \mathrm{ml}$ tube was incubated at $70{ }^{\circ} \mathrm{C}$ for $1 \mathrm{~h}$. One volume $(750 \mu \mathrm{l})$ of $0.1 \mathrm{~mm}$ diameter silica beads (Sigma) previously sterilized by autoclaving was added, and the tube was shakenat maximum speed for $10 \mathrm{~min}$ in a Vibro shaker (Retsch). Polyvinylpolypyrrolidone $(15 \mathrm{mg})$ was added to the tube, which was vortexed and centrifuged for $3 \mathrm{~min}$ at $12,000 \times \mathrm{g}$. After recovery of the supernatant, the pellet was washed with $500 \mu \mathrm{l}$ of TENP (50 mM Tris [pH8], $20 \mathrm{mM}$ EDTA [pH8], $100 \mathrm{mM}$ $\mathrm{NaCl}, 1 \%$ polyvinylpolypyrrolidone) and centrifuged for $3 \mathrm{~min}$ at $12,000 \times \mathrm{g}$, and the new supernatant was added to the first supernatant. The washing step was repeated three times. Pooled supernatants (about $2 \mathrm{ml}$ ) were briefly centrifuged to remove particles and then split into two $2 \mathrm{ml}$ tubes. Nucleic acids were precipitated by the addition of 1 volume of isopropanol for $10 \mathrm{~min}$ at room temperature and centrifuged for $15 \mathrm{~min}$ at $20,000 \times$ g. Pellets were resuspended and pooled in $450 \mu \mathrm{l}$ of $100 \mathrm{mM}$ phosphate buffer (pH8) and $50 \mu \mathrm{l}$ of $5 \mathrm{M}$ potassium acetate. The tube was placed on ice for $90 \mathrm{~min}$ and centrifuged at $16,000 \times \mathrm{g}$ for $30 \mathrm{~min}$. The supernatant was transferred to a new tube containing $20 \mu \mathrm{l}$ of RNase $(1 \mathrm{mg} / \mathrm{ml})$ and incubated at $37{ }^{\circ} \mathrm{C}$ for $30 \mathrm{~min}$. Nucleic acids were precipitated by addition of $50 \mu \mathrm{l}$ of $3 \mathrm{M}$ sodium acetate and $1 \mathrm{ml}$ of absolute ethanol. The tube was incubated for $10 \mathrm{~min}$ at room temperature, and nucleic acids were recovered by centrifugation at $20,000 \times \mathrm{g}$ for $15 \mathrm{~min}$. The DNA pellet was finally washed with $70 \%$ ethanol, dried, and resuspended in $400 \mu \mathrm{l}$ TE buffer. DNA concentration and purity were analyzed by Nanodrop (Thermo). Size distribution (predominantly around $20 \mathrm{~kb}$ ) were estimated by electrophoresis (Additional file 3: Figure S1). Extracted DNA was stored at $-20{ }^{\circ} \mathrm{C}$ until use.

Sequences involving V3 and V4 16S rDNA hypervariable regions were amplified by TranStart FastPfu DNA Polymerase (TransGen Biotech, China) using the following primers (5' to 3'): 341 F-CCTACGGGNGGCWGCAG, 805R-GACTACHVGGGTATCTAATCC. PCR products were analyzed and separated by electrophoresis on $2 \%$ agarose gel (containing SYB green), then purified with Qiagen Gel Extraction Kit (Qiagen, Germany). Sequencing libraries were generated using TruSeq DNA PCR manufacturer's instructions and index codes were added. The library was sequenced and analyzed using an Illumina HisSeq2500 platform by Shanghai Tai Chang gene technology co., LTD., China

\section{Statistical analysis}

All data were analyzed using Prism 5.0 (GraphPad Software Inc., CA, USA). Results were represented as mean \pm SEM. Two-way analysis of variance (ANOVA) followed by the Turkey multiple-comparison test was used to determine statistical difference between experimental groups. Results were considered significant at $P<0.05$.

\section{Results}

Routine parameters of mice in diverse dietary groups

There was no significant difference in initial body weight (BW) among four groups. However, after 6 weeks feeding, the final BW in AF/CO group was significantly decreased, compared with that in paired $\mathrm{PF} / \mathrm{CO}$ group $(P<0.01)$ or AF/FO group $(P<0.01)$. The final BW in $\mathrm{AF} / \mathrm{FO}$ showed no change compared with PF/FO. These results demonstrated that flaxseed oil maintained the BW during chronic ethanol feeding. Liver weight in AF group (AF/ 
$\mathrm{CO}$ group and AF/FO group) was significantly elevated comparing to that in PF group (PF/CO group and PF/FO group) (Table 1). Similarly, the ratio of liver-to-body weight in alcohol exposure group regardless of dietary fat was significantly increased compared with that in no ethanol pair-fed group. In addition, the plasma AST and ALT levels in AF/CO group were significantly elevated by 2.5fold $(185.9 \pm 13.3$ vs. $74.8 \pm 8.6)$ and 2-fold $(104.8 \pm 11.4$ vs. $52.6 \pm 5.9)$ compared with that in pair-fed $\mathrm{PO} / \mathrm{CO}$ group, respectively. However, these AST and ALT elevations in $\mathrm{AF} / \mathrm{CO}$ group were effectively suppressed by dietary FO administration in AF/FO group (185.9 \pm 13.3 vs. $109.7 \pm 7.2,104.8 \pm 11.4$ vs. $75.2 \pm 6.1$ ) (Table 1$)$.

\section{Dietary FO attenuated hepatic histopathological injury and reduced plasma LPS levels}

According to HE staining for liver in diverse groups, hepatic fatty change, necrosis and inflammation were serious in chronic alcohol feeding group (AF/CO), whereas longterm dietary FO distinctly alleviated the alcohol-induced hepatic histopathological injury (Fig. 1a).

Plasma LPS in AF/FO group was significantly decreased compared with AF/CO group $(P<0.0001)$, but still higher than PF/CO or PF/FO group (Fig. 1b), demonstrating that dietary FO possessed ability to attenuated LPS generation from Gram-negative pathogenic bacteria.

\section{Dietary FO reduced plasma inflammatory cytokine levels in ALD}

After chronic ethanol feeding, we found obvious elevated plasma TNF- $\alpha$, IL-1 $\beta$, IL- 6 and IL-10 in AF/CO and AF/ FO groups compared with these cytokines in pair-fed group (Fig. 2). However, dietary FO attenuated ethanol-inducing abnormal elevated TNF- $\alpha$ concentration, compared with that in PF control group $(P=0.0095$, Fig. 2a). Similarly, plasma IL-1 $\beta \quad(P=0.007$, Fig. $2 \mathrm{~b})$ and IL-6 $(P<0.0001$, Fig. 2c) levels in AF/FO were also significantly reduced in comparison with those two cytokines in $\mathrm{AF} / \mathrm{CO}$ group. It showed no significant difference in plasma IL-10 level between $\mathrm{AF} / \mathrm{CO}$ and $\mathrm{AF} / \mathrm{FO}$ groups $(P=0.3229$, Fig. $2 \mathrm{~d})$.

\section{Dietary FO reduced liver inflammatory cytokine levels in ALD}

We detected the cytokine production in liver tissue and also found elevated TNF- $\alpha$, IL- $1 \beta$, IL- 6 and IL-10 in AF group compared with PF group. Similarly, TNF$\alpha(p<0.001$, Fig. 3a), IL-1 $\beta(P=0.0021$, Fig. 3b $)$ and IL-6 $(P=0.0022$, Fig. 3c) levels in AF/FO group were significantly decreased compared with those three cytokines in $\mathrm{AF} / \mathrm{CO}$ group. It showed also no significant difference in IL-10 level in supplementary FO group during chronic ethanol feeding $(P=0.1635$, Fig. 3d).

\section{Dietary FO modulated gut microbiota in ALD}

Gut microbiota have been increasingly thought to play a critical role in ALD development in mice and humans $[3,14-18]$. To investigate whether the observed differences in liver inflammation among $\mathrm{AF} / \mathrm{CO}, \mathrm{AF} / \mathrm{FO}$ and those PF groups were associated with the difference in the intestinal microbiota, we performed fecal metagenomic analysis. Rationality of sequencing data was evaluated by rarefaction curve (Additional file 4: Figure S2). It was observed that the rarefaction curve tended to be flat when the sequence number increased to 20,000, indicating that the amount of sequencing data was reasonable.

The overall bacterial community structure was analyzed using unweighted UniFrac (Pcoa) (Fig. 4) and weighted distance matrices (NMDS) (Additional file 5: Figure S3). Pcoa showed that chronic alcohol consumption induced an obvious difference in terms of species in fecal samples compared with pair-fed control feeding (Fig. 4a and b). There's no obvious change in terms of species between $\mathrm{AF} / \mathrm{CO}$ group and AF/FO group (Fig. 4c). Interestingly, during normal liquid feeding, supplementary FO seemingly altered the fecal species compared with $\mathrm{CO}$ feeding (Fig. 4d). Similar results from NMDS analysis were obtained (Additional file 5: Figure S3).

At phylum level, the proportion of Firmicutes was notably increased in alcohol feeding groups compared with those in the PF groups $(P=0.0159$, Fig. 5a). Meanwhile, there's no change between $\mathrm{AF} / \mathrm{FO}$ and $\mathrm{AF} / \mathrm{CO}$ groups $(P=0.8385$, Fig. 5a). Bacteroidetes accounted for more than half of proportion in diverse administration groups

Table 1 Routine parameters of mice in diverse dietary groups in ALD

\begin{tabular}{lccccccc}
\hline Measurements & PF/CO & AF/CO & PF/FO & AF/FO & \multicolumn{3}{l}{ Two-way ANOVA } \\
\cline { 5 - 7 } & & & & & Ethanol & Oil & Interaction \\
\hline Body weight, g & $26.15 \pm 0.27$ & $23.99 \pm 0.29$ & $26.34 \pm 0.33$ & $26.57 \pm 0.28$ & $<0.0001$ & 0.0019 & 0.0002 \\
Liver weight, g & $0.89 \pm 0.03$ & $1.25 \pm 0.04$ & $1.00 \pm 0.02$ & $1.44 \pm 0.04$ & $<0.0001$ & $<0.0001$ & 0.2722 \\
LW/BW, \% & $3.40 \pm 0.11$ & $5.21 \pm 0.14$ & $3.80 \pm 0.06$ & $5.42 \pm 0.14$ & $<0.0001$ & $<0.0001$ & 0.0027 \\
AST, U/L & $74.8 \pm 8.6$ & $185.9 \pm 13.3$ & $68.4 \pm 6.7$ & $109.7 \pm 7.2$ & $<0.0001$ & $<0.0001$ & $<0.0001$ \\
ALT, U/L & $52.6 \pm 5.9$ & $104.8 \pm 11.4$ & $47.6 \pm 8.2$ & $75.2 \pm 6.1$ & $<0.0001$ & $<0.0001$ & $<0.0001$ \\
\hline
\end{tabular}




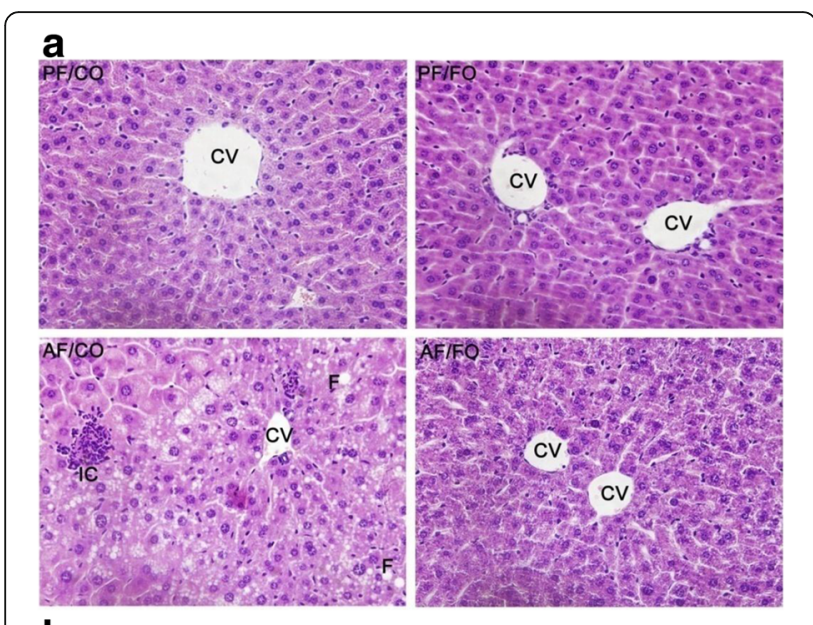

b

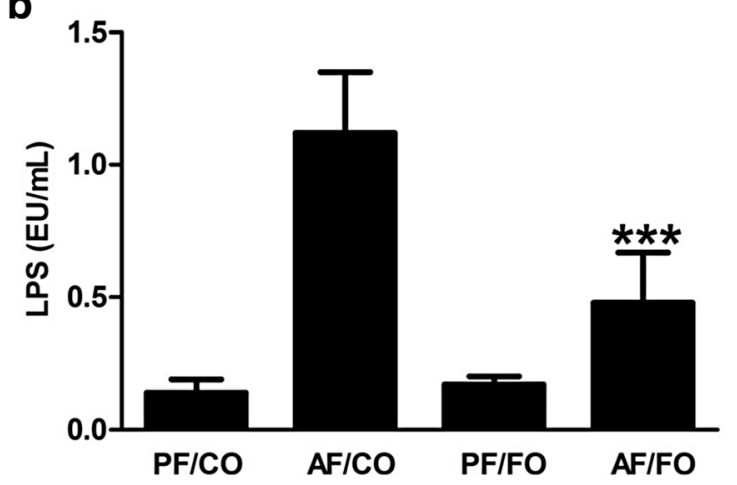

Fig. 1 Effects of different dietary oil profile on liver injury and endotoxemia in ALD. a: Representative images of hepatic hemaatoxylin and eosin (H\&E) staining. b: Plasma lipopolysaccharide (LPS) levels. Data are expressed as mean \pm SEM. ${ }^{*} P<0.05,{ }^{* *} P<0.001,{ }^{* * *} P<0.0001$. Original magnification, $\times 200$ (A). CV, central vein; F, fatty change; IC, inflammatory cells and decreased in $\mathrm{AF} / \mathrm{CO}$ group in comparison with other three groups but with no significant difference. The proportion of Proteobacteria showed no alteration in chronic consumption of alcohol compared with nonethanol controls. The proportion of Proteobacteria in AF/ FO group was significantly lower than that in $\mathrm{AF} / \mathrm{CO}$ group $(0.074 \pm 0.009$ vs. $0.117 \pm 0.003, P<0.0001)$ or $\mathrm{PF} /$ FO group $(0.074 \pm 0.009$ vs. $0.124 \pm 0.009, P<0.0001)$. Taken together, our data revealed that under this experimental condition a combination of ethanol and dietary FO (AF/FO) had a major effect on Proteobacteria but with limited effects on Bacteriodetes and Firmicutes.

At genus level, we found Porphyromonadaceae was the most prevalent genus in the control groups (PF/CO and $\mathrm{PF} / \mathrm{FO}$ ) and obviously reduced in dietary alcohol administration groups $(P<0.0001$, Fig. $5 b)$. Moreover, the proportion of Porphyromonadaceae in AF/FO group showed lower than that in $\mathrm{AF} / \mathrm{CO}$ group but without significance $(0.176 \pm 0.026$ vs. $0.146 \pm 0.013, P=0.0503)$. In contrast, Parabacteroides was sharply elevated in the $\mathrm{AF})$ groups (AF/CO and $\mathrm{AF} / \mathrm{FO})$ compared with the control groups $(P=0.0211$, Fig. 5b). Additionally, Parasutterella was the second prevalent genus in each group. Alcohol administration induced a significant reduction of Parasutterella in comparison to that in the control groups $(P=0.0005)$. Collectively, our genus results indicating that chronic alcohol consumption obviously altered the initial proportion of genus components, mainly including Porphyromonadaceae, Parabacteroides and Parasutterella.

Furthermore, heatmap also showed that dietary FO (AF/FO) had a major effect on Proteobacteria, with
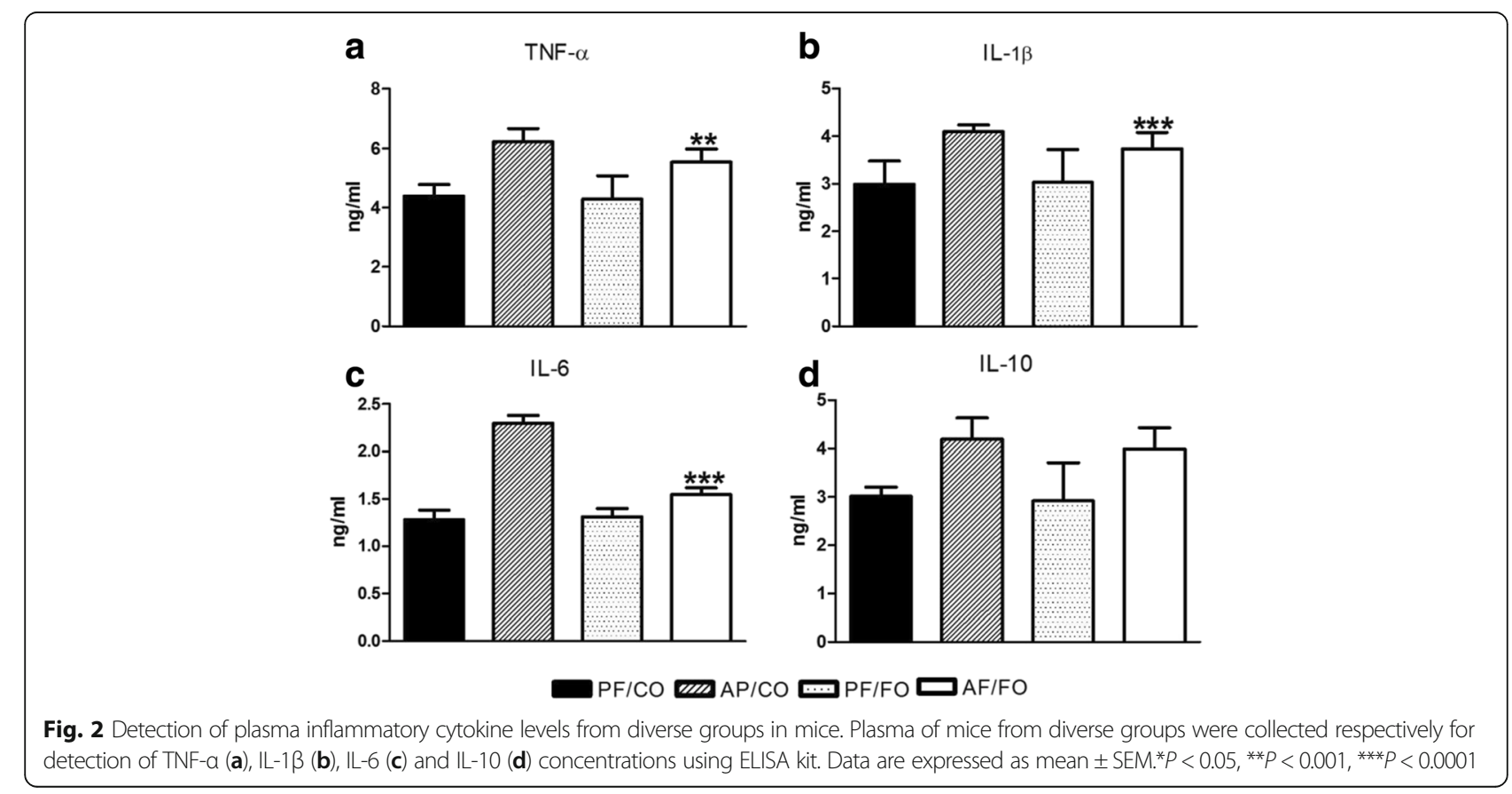


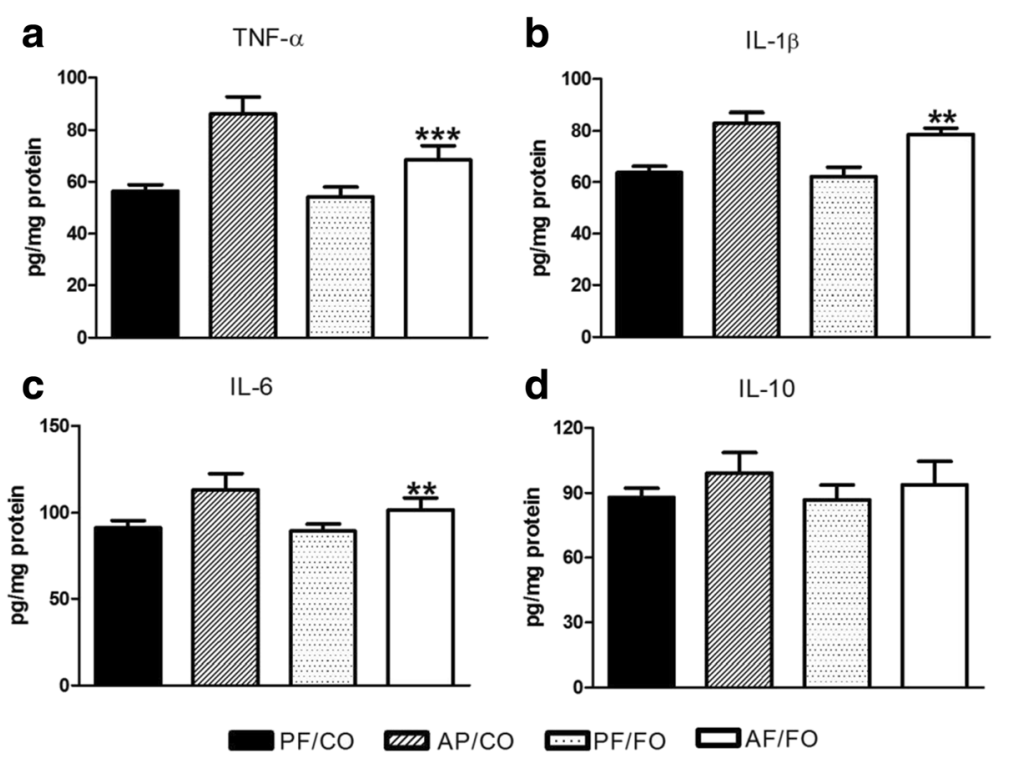

Fig. 3 Detection of hepatic inflammatory cytokine levels from diverse groups in mice. Liver tissue of mice from diverse groups were collected respectively for detection of TNF-a (a), IL-1 3 (b), IL-6 (c) and IL-10 (d) concentrations using ELISA kit. Data are expressed as mean \pm SEM.*P $<0.05$, ${ }^{*} P<0.001$, ${ }^{* * *} P<0.0001$
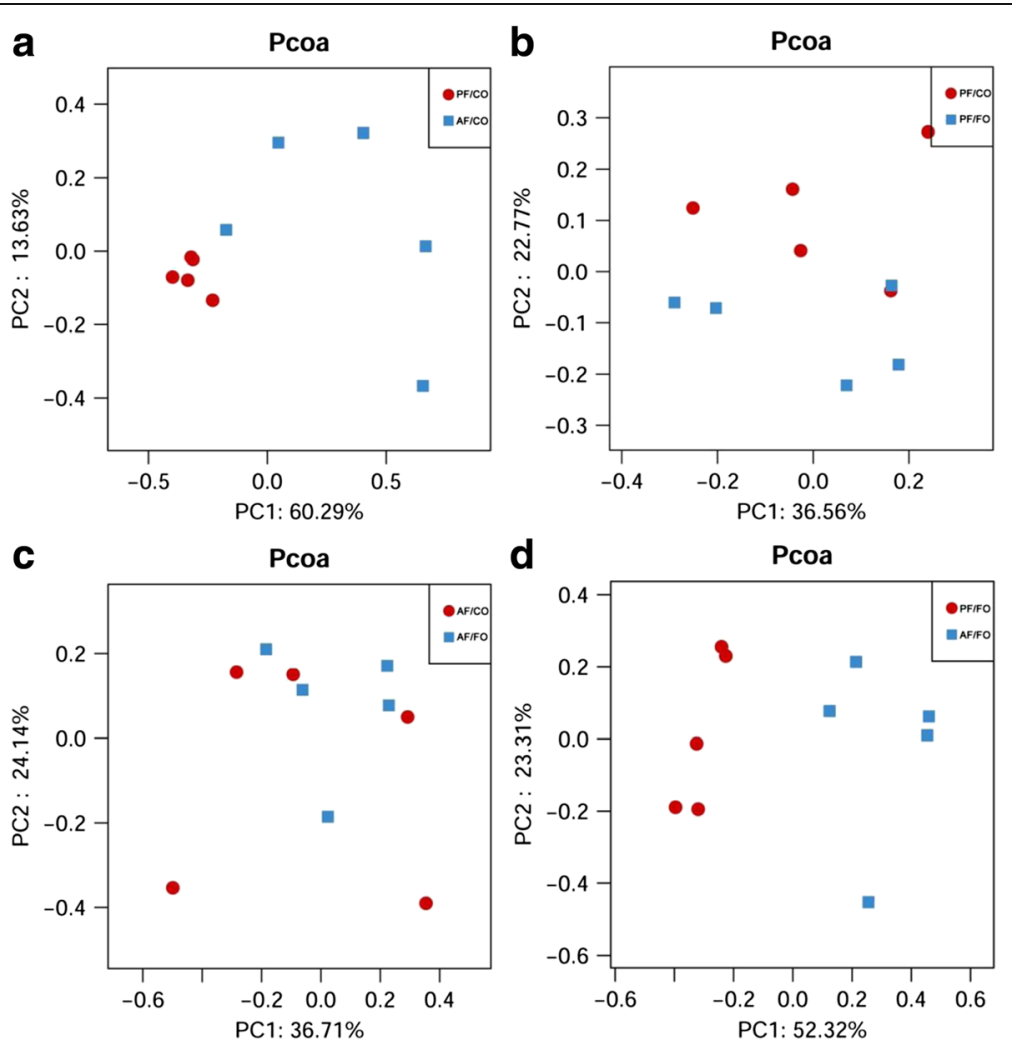

Fig. 4 PCOA analysis showing difference in terms of species in fecal samples. Beta diversity was on weighted UniFrac. a: PF/CO vs. AF/CO; b: PF/ $\mathrm{CO}$ vs. PF/FO; c: AF/CO vs. AF/FO; d: PF/FO vs. AF/FO 

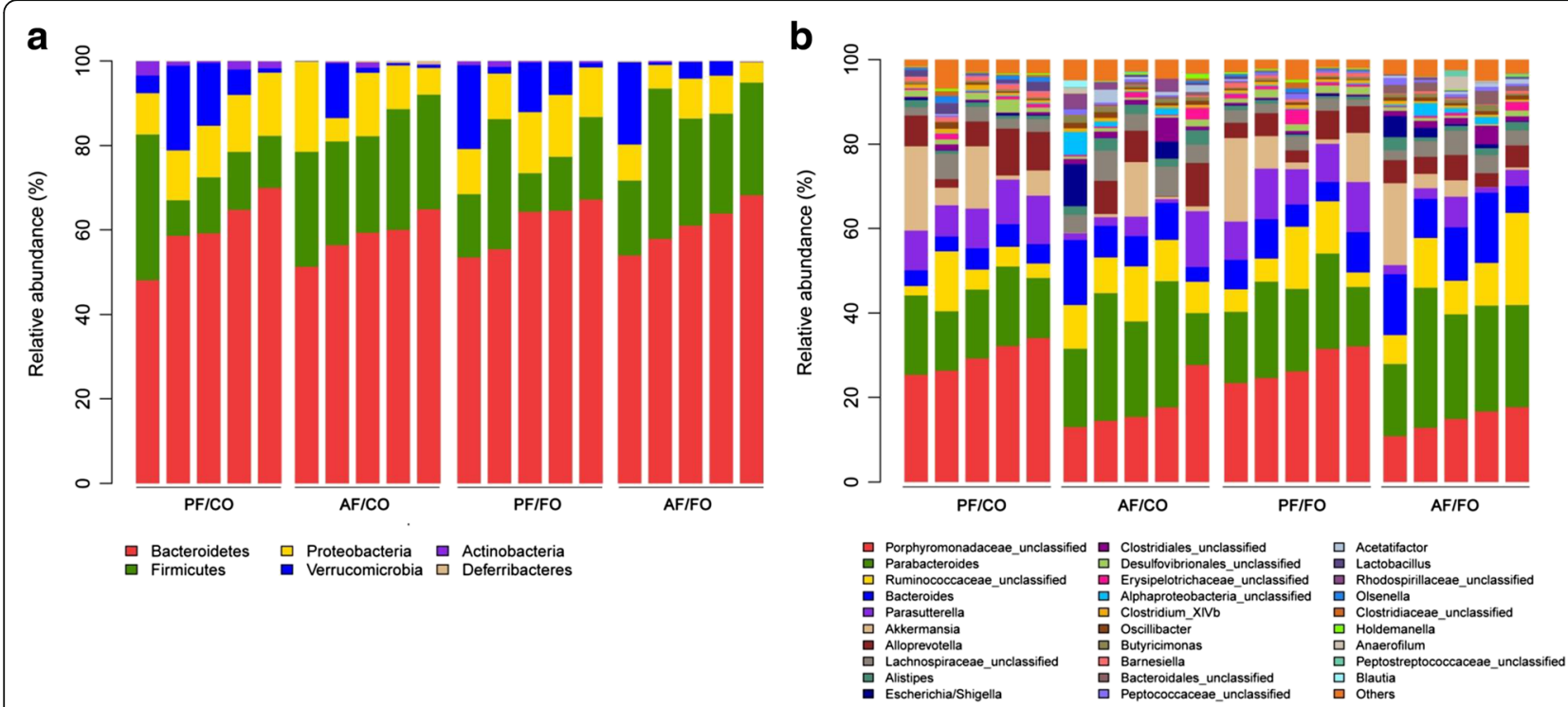

Fig. 5 Relative abundance of microbial species at the phylum and genus levels in the feces of mice. a: The phylum analysis; b: The genus analysis

limited effects on Bacteriodetes and Firmicutes. Moreover, many other tiny bacteria showed obvious difference between AF and PF groups, such as Barnesiella, Psychrobacter, Deltaproteobacteria, Acinetobacter, Flavonifractor, and Lactococcus (Fig. 6a). However, diverse dietary oil had a less effect of on the influence of these seldom bacteria proportion (Fig. 6b).

\section{Discussion}

In the present study, we investigated the efficacy of long-term dietary FO for chronic ALD. By in vivo 6weeks treatment of ALD in mice, our study demonstrated that supplementary FO showed more effective in reduction of hepatic damage, suggesting that this inexpensive interventions exhibited preventive and therapeutic potential. Our further study revealed that this effective treatment may associated with altered gut microbiota and the decrease of liver inflammation.

Numerous studies indicated that alcohol exposure significantly reduced final BW in chronic ALD $[3,9,11,19]$. In this study, we also found that $\mathrm{BW}$ was lower in $\mathrm{AF} / \mathrm{CO}$ group, although the caloric intake was identical among all groups. Dietary FO efficiently improved the final BW in ALD compared with AF/CO, indicating that FO may positively affect nutrients absorption and efficiency of calorie utilization in gastrointestinal tract in ALD. Liver weight and relative liver weights in AF group regardless of dietary oil significantly increased, which was consistent with previous reports [9], suggesting that substituting $\mathrm{FO}$ for $\mathrm{CO}$ in chronic ethanol intake had no effect on liver weight.

In this study, we found abnormal elevated plasma ALT and AST levels in AF/CO group, indicating alcohol induced liver injury [9]. Significant reductions of plasma ALT and AST in AF/FO group revealed that supplementary FO alleviated liver damage caused by chronic ethanol feeding. Similarly, dietary fish oil, rich in long-chain n-3 polyunsaturated fatty acids, mainly eicosapentaenoic acid (EPA) and docosahexaenoic acid (DHA), has showed also the ability to attenuate liver injury by reducing ALT and AST levels in ALD [9, 17]. Inexpensive dietary FO-derived ALA, served as a precursor for the synthesis of EPA and DHA, can converse to EPA and DHA in the blood and tissues [20].

LPS, a trigger for hepatic inflammation in ALD, translocates to liver via portal vein and binds to TLR-4 of antigen presenting cells (APCs) to induce inflammatory immune response and finally cause chronic hepatitis [21, 22]. In this study, plasma LPS in AF/FO group was obviously decreased, demonstrating that dietary FO may decrease gut permeability and reduce LPS translocation from intestines to the liver and systematic circulation in ALD, which contributed to the reduction of inflammatory response in the liver. This attenuation may be associated with intestinal innate immune system and the underlying mechanism needs to be further researched [23].

Activation of Kupffer cells and neutrophils induces oxidative stress and produces inflammatory cytokines, such as TNF- $\alpha$, IL- $1 \beta$ and IL- 6 that cause apoptosis and necrosis of hepatocytes and consequently result in liver injury $[9,24,25]$. Our results showed that TNF- $\alpha$, IL- $1 \beta$ and IL6 levels of plasma and liver tissue in AF/FO group were significantly decreased, demonstrating that dietary FO alleviated hepatic inflammation via anti-inflammatory cytokines. IL-10 is an anti-inflammatory cytokine released by Kupffer cells and monocytes [26, 27]. But in this study, we 

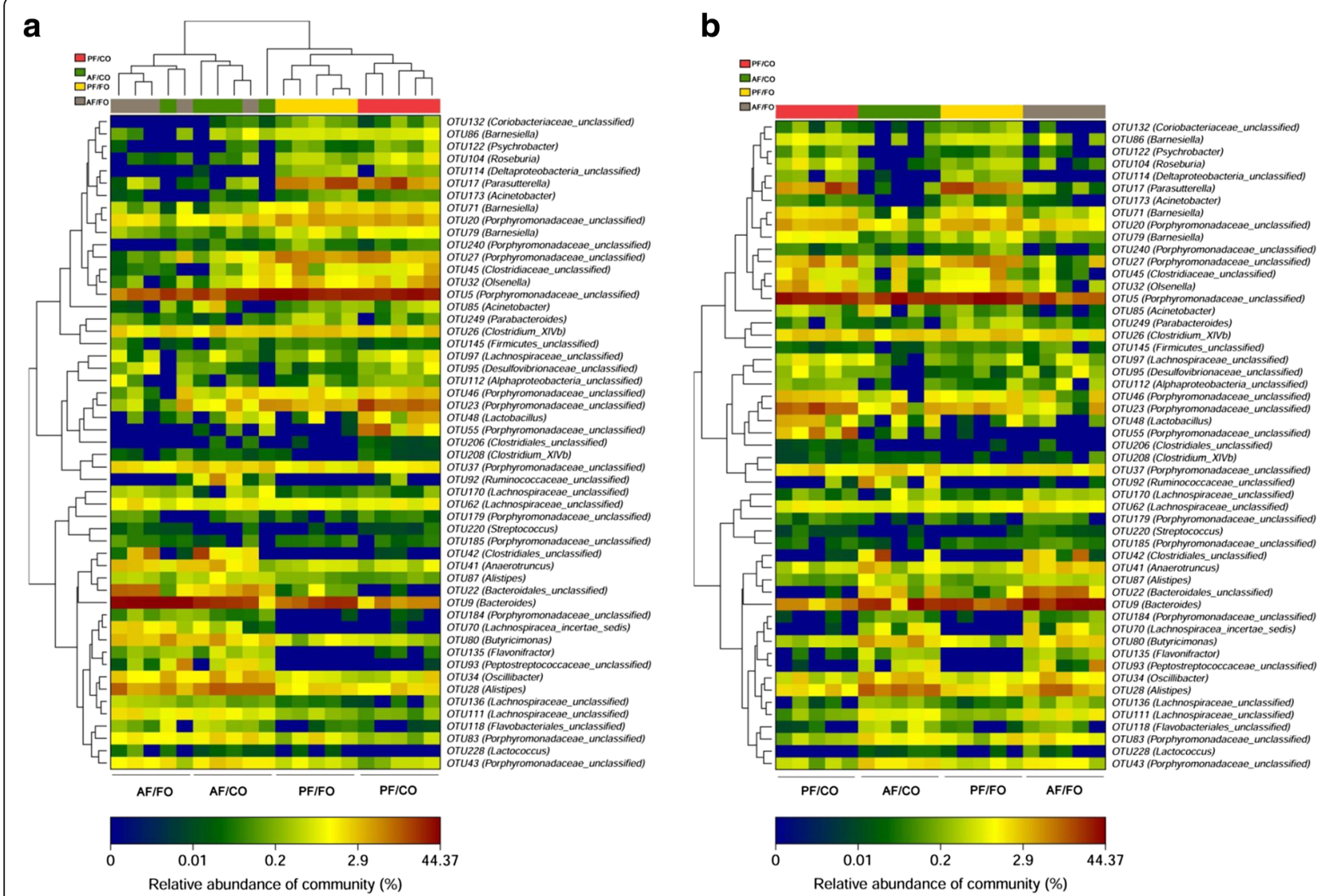

Fig. 6 Heatmap analysis of microbial community composition in the feces of mice. a: alcohol-fed (AF) vs. pair-fed (PF); b: flaxseed oil (FO) vs. corn oil (CO)

found IL-10 showed no difference among all groups, which was not paralleled with previous study [9]. We speculated that IL-10 maybe play a complicated role in imbalance between regulation of pro- and anti- inflammatory mediators during chronic ethanol exposure. Additionally, regulatory immune cells especially regulatory $\mathrm{T}$ lymphocytes (Tregs) [28], which play a critical role in regulation of proinflammation to keep maintain immune balance in ALD [29, 30], need to be investigated in our further study.

Gut micobiota dysbiosis is thought to play a crucial role in the pathogenesis of ALD [6,31,32]. In this study, at phylum level, Bacteriodetes and Firmicutes were the most dominant in all four groups, which were paralleled with previous studies [12,33]. The proportion of Firmicutes was notably increased in alcohol feeding groups compared with the PF groups, which were in agreement with previous studies [3, 32]. Our results showed decreased Bacteriodetesand higher Proteobacteriain alcohol intake group (AF/CO), which were responsible for gut dysbiosis as recently described in human and animal studies $[3,18]$. Importantly, dietary FO notably reduced the proportion of Proteobacteria in chronic alcohol consumption, revealing that dietary FO may attenuate gut dysbiosis presumably by modulating gut Proteobacteria. Exact mechanism(s) underlying these effects remain to be determined.

At the genus level, decreased gut Porphyromonadaceae and inversely elevated Parabacteroides were found in chronic alcohol administration. Porphyromonadaceae was negatively correlated with TNF- $\alpha$ expression in the liver in ALD [34], which was paralleled with our result and the decrease of gut Porphyromonadaceae may benefit for aggravation of the liver inflammation. Elevated Parabacteroidesin $\mathrm{AF} / \mathrm{FO}$ group was also involved in the prevention of hepatic inflammation in ALD as previously described [34]. Our results showed that alcohol administration induced a significant reduction of Parasutterella in comparison to the control groups. The physiological role of Parasutterella is much less understood. Taken together, the exact role of microbiota is complicated and still largely unknown.

\section{Conclusions}

This study highlighted that dietary FO ameliorates alcoholic liver disease via anti-inflammation and modulating gut microbiota in mice, suggesting that it can potentially serve as inexpensive interventions for the prevention and treatment of ALD. 


\section{Additional files}

Additional file 1: Table S1. Compositions of the modified LieberDeCarli liquid diets. PF/CO, pair-fed with corn oil; AF/CO, alcohol-fed with corn oil; PF/FO, pair-fed with flaxseed oil; AF/FO, alcohol-fed with flaxseed oil (DOCX $12 \mathrm{~kb})$

Additional file 2: Table S2. Fatty acid composition (\%) of dietary fats contained in liquid diets. (DOCX $12 \mathrm{~kb}$ )

Additional file 3: Figure S1. Size distribution (predominantly around $20 \mathrm{~kb}$ ) was estimated by electrophoresis. (DOCX 62 kb)

Additional file 4: Figure S2. Rationality of sequencing data was evaluated by rarefaction curve. It was observed that the rarefaction curve tended to be flat when the sequence number increased to 20,000 , indicating that the amount of sequencing data was reasonable. (DOCX $115 \mathrm{~kb}$ )

Additional file 5: Figure S3. NMDS analysis showed the difference in terms of species in fecal samples. Beta diversity was analyzed on unweighted Unifrac. A: PF/CO vs. AF/CO; B: PF/CO vs. AF/FO; C: AF/CO vs. AF/FO; D: PF/CO vs. PF/FO. (DOCX $136 \mathrm{~kb}$ )

Additional file 6: Datasets for Figures S1-S6. (ZIP 258 kb)

\section{Abbreviations}

AF: Alcohol-fed; ALA: a-linolenic acid; ALD: Alcoholic liver disease; ALT: Alanine aminotransferase; APCs: Antigen presenting cells; AST: Aspartate aminotransferase; BW: Body weight; CO: Corn oil; DHA: Docosahexaenoic acid; EDTA: Ethylene diamine tetraacetic acid; ELISA: Enzyme linked immunosorbent assay; EPA: Eicosapentaenoic acid; FO: Flaxseed oil; HE: Hematoxylin-eosin; IL: Interleukin; LPS: Lipopolysaccharide; PF: Pair-fed; PUFA: Polyunsaturated fatty acids; TLR-4: Toll-like receptor-4; TNF-a: Tumor necrosis factor; Tregs: Regulatory T lymphocytes

\section{Acknowledgements}

Not applicable.

\section{Funding}

This work was supported by the special funds for Forestry Public Welfare Scientific Research Projects (No. 201404718), China.

\section{Availability of data and materials}

The Additional file 6: used and analysed during the current study are available from the corresponding author on reasonable request.

\section{Authors' contributions}

LYJ and ZXX designed and wrote the paper. ZXX, WH, YPP, FH and SLW performed research. All authors have read and approved the final manuscript.

\section{Competing interests}

The authors declare that they have no competing interests. The funding body played no role in the design of the study and collection, analysis, and interpretation of data and in writing the manuscript.

\section{Consent for publication}

Not applicable.

\section{Ethics approval and consent to participate}

All animal experiments were approved by the Ethics Committee of Ningxia Medical University (document no. LA2015-114), and carried out in accordance with the 2011 revised form of The Guide for the Care and Use of Laboratory Animals published by the U.S. National Institutes of Health.

\section{Author details}

${ }^{1}$ College of Biological Sciences and Biotechnology, Beijing Forestry University, Qinghua Donglu No35, Haidian District, Beijing 100083, China. ${ }^{2}$ Ningxia Medical University, Yinchuan 750004, Ningxia, China.
Received: 15 January 2017 Accepted: 13 February 2017

Published online: 22 February 2017

\section{References}

1. Rehm J, Mathers C, Popova S, Thavorncharoensap M, Teerawattananon Y, Patra J. Global burden of disease and injury and economic cost attributable to alcohol use and alcohol-use disorders. Lancet. 2009:373:2223-33.

2. Di MA. Therapy of digestive disorders: a companion to sleisenger and Fordtran's gastrointestinal and liver disease. Gastroenterology. 2000;118:1275-6.

3. Kirpich IA, Petrosino J, Ajami N, Feng W, Wang Y, Liu Y, Beier JI, Barve SS, Yin X, Wei $X$, et al. Saturated and unsaturated dietary fats differentially modulate ethanol-induced changes in Gut microbiome and metabolome in a mouse model of alcoholic liver disease. Am J Pathol. 2016;186:765-76.

4. Allampati S, Mullen KD. Long-term management of alcoholic liver disease. Clin Liver Dis. 2016;20:551-62.

5. Altamirano J, Bataller R. Alcoholic liver disease: pathogenesis and new targets for therapy. Nat Rev Gastroenterol Hepatol. 2011;8:491-501.

6. Szabo G. Gut-liver axis in alcoholic liver disease. Gastroenterology. 2015;148:30-6.

7. Szabo G, Bala S. Alcoholic liver disease and the gut-liver axis. World J Gastroenterol. 2010;16:1321-9.

8. M CCC, N LL, C MF, J LG, D A, C G, G C, S HP, C M, F SM, et al. Comparing the effects of acute alcohol consumption in germ-free and conventional mice: the role of the gut microbiota. BMC Microbiol. 2014;14:240.

9. Chen JR, Chen YL, Peng HC, Lu YA, Chuang HL, Chang HY, Wang HY, Su YJ, Yang SC. Fish Oil reduces hepatic injury by maintaining normal intestinal permeability and microbiota in chronic ethanol-Fed rats. Gastroenterol Res Pract. 2016;2016:4694726.

10. Shapiro H, Tehilla M, Attal-Singer J, Bruck R, Luzzatti R, Singer P. The therapeutic potential of long-chain omega-3 fatty acids in nonalcoholic fatty liver disease. Clin Nutr. 2011;30:6-19.

11. Wang M, Zhang XJ, Feng K, He C, Li P, Hu YJ, Su H, Wan JB. Dietary alphalinolenic acid-rich flaxseed oil prevents against alcoholic hepatic steatosis via ameliorating lipid homeostasis at adipose tissue-liver axis in mice. Sci Rep. 2016;6:26826.

12. Zhou Z, Wang Y, Jiang Y, Diao Y, Strappe P, Prenzler P, Ayton J, Blanchard C. Deep-fried oil consumption in rats impairs glycerolipid metabolism, gut histology and microbiota structure. Lipids Health Dis. 2016;15:86.

13. Godon JJ, Zumstein E, Dabert P, Habouzit F, Moletta R. Molecular microbial diversity of an anaerobic digestor as determined by small-subunit rDNA sequence analysis. Appl Environ Microbiol. 1997;63:2802-13.

14. Mutlu E, Keshavarzian A, Engen P, Forsyth CB, Sikaroodi M, Gillevet P. Intestinal dysbiosis: a possible mechanism of alcohol-induced endotoxemia and alcoholic steatohepatitis in rats. Alcohol Clin Exp Res. 2009:33:1836-46.

15. Gabbard SL, Lacy BE, Levine GM, Crowell MD. The impact of alcohol consumption and cholecystectomy on small intestinal bacterial overgrowth. Dig Dis Sci. 2014;59:638-44.

16. Bull-Otterson L, Feng W, Kirpich I, Wang Y, Qin X, Liu Y, Gobejishvili L, JoshiBarve S, Ayvaz T, Petrosino J, et al. Metagenomic analyses of alcoho induced pathogenic alterations in the intestinal microbiome and the effect of Lactobacillus rhamnosus GG treatment. PLoS One. 2013;8:e53028.

17. Chen $P$, Torralba M, Tan J, Embree M, Zengler $K$, Starkel $P$, van Pijkeren JP, DePew J, Loomba R, Ho SB, et al. Supplementation of saturated long-chain fatty acids maintains intestinal eubiosis and reduces ethanol-induced liver injury in mice. Gastroenterology. 2015;148:203-14. e216.

18. Mutlu EA, Gillevet PM, Rangwala H, Sikaroodi M, Naqvi A, Engen PA, Kwasny M, Lau CK, Keshavarzian A. Colonic microbiome is altered in alcoholism. Am J Physiol Gastrointest Liver Physiol. 2012;302:G966-978.

19. Lieber CS. Hepatic, metabolic and toxic effects of ethanol: 1991 update. Alcohol Clin Exp Res. 1991;15:573-92.

20. Taylor CG, Noto AD, Stringer DM, Froese S, Malcolmson L. Dietary milled flaxseed and flaxseed oil improve N-3 fatty acid status and do not affect glycemic control in individuals with well-controlled type 2 diabetes. J Am Coll Nutr. 2010:29:72-80.

21. Parlesak A, Schafer C, Schutz T, Bode JC, Bode C. Increased intestinal permeability to macromolecules and endotoxemia in patients with chronic alcohol abuse in different stages of alcohol-induced liver disease. J Hepatol. 2000;32:742-7.

22. Fukui H, Brauner B, Bode JC, Bode C. Plasma endotoxin concentrations in patients with alcoholic and non-alcoholic liver disease: reevaluation with an improved chromogenic assay. J Hepatol. 1991;12:162-9. 
23. Thaiss CA, Zmora N, Levy M, Elinav E. The microbiome and innate immunity Nature. 2016;535:65-74.

24. Gao B. Hepatoprotective and anti-inflammatory cytokines in alcoholic liver disease. J Gastroenterol Hepatol. 2012;27 Suppl 2:89-93.

25. Neuman MG, Maor Y, Nanau RM, Melzer E, Mell H, Opris M, Cohen L, Malnick S. Alcoholic liver disease: role of cytokines. Biomolecules. 2015;5:2023-34.

26. Byun JS, Suh YG, Yi HS, Lee YS, Jeong WI. Activation of toll-like receptor 3 attenuates alcoholic liver injury by stimulating Kupffer cells and stellate cells to produce interleukin-10 in mice. J Hepatol. 2013;58:342-9.

27. de Waal MR, Abrams J, Bennett B, Figdor CG, de Vries JE. Interleukin 10(IL10) inhibits cytokine synthesis by human monocytes: an autoregulatory role of IL-10 produced by monocytes. J Exp Med. 1991;174:1209-20.

28. Hawrylowicz CM, O'Garra A. Potential role of interleukin-10-secreting regulatory T cells in allergy and asthma. Nat Rev Immunol. 2005;5:271-83.

29. Chen RC, Xu LM, Du SJ, Huang SS, Wu H, Dong JJ, Huang JR, Wang XD, Feng WK, Chen YP. Lactobacillus rhamnosus GG supernatant promotes intestinal barrier function, balances Treg and TH17 cells and ameliorates hepatic injury in a mouse model of chronic-binge alcohol feeding. Toxicol Lett. 2016;241:103-10.

30. Kasztelan-Szczerbinska B, Surdacka A, Celinski K, Rolinski J, Zwolak A, Miacz S, Szczerbinski M. Prognostic significance of the systemic inflammatory and immune balance in alcoholic liver disease with a focus on gender-related differences. PLoS One. 2015;10:e0128347.

31. Cresci GA. The gut microbiome: a new frontier for alcohol investigation. Alcohol Clin Exp Res. 2015;39:947-9.

32. Ferrere G, Wrzosek L, Cailleux F, Turpin W, Puchois V, Spatz M, Ciocan D, Rainteau D, Humbert L, Hugot C, et al. Fecal microbiota manipulation prevents dysbiosis and alcohol-induced liver injury in mice. J Hepatol. 2016. doi:10.1016/j.jhep.2016.11.008. In press.

33. Qin J, Li R, Raes J, Arumugam M, Burgdorf KS, Manichanh C, Nielsen T, Pons $\mathrm{N}$, Levenez F, Yamada T, et al. A human gut microbial gene catalogue established by metagenomic sequencing. Nature. 2010;464:59-65.

34. Neyrinck AM, Etxeberria U, Taminiau B, Daube G, Van Hul M, Everard A, Cani $P D$, Bindels LB, Delzenne NM. Rhubarb extract prevents hepatic inflammation induced by acute alcohol intake, an effect related to the modulation of the gut microbiota. Mol Nutr Food Res. 2017;61:1500899.

\section{Submit your next manuscript to BioMed Central and we will help you at every step:}

- We accept pre-submission inquiries

- Our selector tool helps you to find the most relevant journal

- We provide round the clock customer support

- Convenient online submission

- Thorough peer review

- Inclusion in PubMed and all major indexing services

- Maximum visibility for your research

Submit your manuscript at www.biomedcentral.com/submit

) Biomed Central 